\title{
Conventional and alternative sites for left ventricular assist device inflow and outflow cannula placement
}

\author{
Antonio Loforte ${ }^{1}$, Tomaso Bottio ${ }^{2}$, Matteo Attisani ${ }^{3}$, Sofia Martin Suarez ${ }^{1}$, Vincenzo Tarzia ${ }^{2}$, \\ Marco Pocar ${ }^{3}$, Luca Botta ${ }^{1}$, Gino Gerosa ${ }^{2}$, Mauro Rinaldi ${ }^{3}$, Davide Pacini ${ }^{1}$ \\ ${ }^{1}$ Division of Cardiac Surgery, IRCCS Azienda Ospedaliero-Universitaria di Bologna, Bologna, Italy; ${ }^{2}$ Department of Cardiac Surgery, Centro \\ Gallucci, Padua University, Padua, Italy; ${ }^{3}$ Department of Cardiac Surgery, Città della Salute e della Scienza, Turin University, Turin, Italy \\ Correspondence to: Dr. Antonio Loforte, MD, PhD. Cardiac Surgeon, Department of Cardiac Surgery, S. Orsola Hospital, ALMA Mater Studiorum \\ University of Bologna, Via Massarenti n.9, 40138 Bologna, Italy. Email: antonioloforte@yahoo.it.
}

\begin{abstract}
Left ventricular assist device (LVAD) therapy is a well accepted and effective strategy to treat advanced heart failure. The miniaturized third-generation centrifugal pumps HeartWare HVAD (Medtronic, Dublin, Ireland) and HeartMate 3 (Abbott, Illinois, USA) are the two most commonly implanted systems in the contemporary era. Their design has allowed clinicians to pioneer several alternate and less invasive implantation techniques to tackle a broad spectrum of clinical scenarios. A brief review and discussion of alternative surgical techniques for both inflow and outflow cannula insertion, in the contemporary LVAD surgery era, are herein reported.
\end{abstract}

Keywords: Left ventricular assist device (LVAD); less invasive cardiac surgery; inflow cannula; outflow cannula; biventricular assist device (BiVAD)

Submitted Nov 04, 2020. Accepted for publication Mar 14, 2021.

doi: 10.21037/acs-2020-cfmcs-28

View this article at: http://dx.doi.org/10.21037/acs-2020-cfmcs-28

\section{Introduction}

Left ventricular assist device (LVAD) therapy is a well accepted and effective strategy to treat advanced heart failure (1-3). Advances in LVAD pump technology, evolving from bulky pulsatile devices to smaller continuous-flow (CF) pumps, combined with a better understanding of patient management, have allowed progressive improvement in long-term outcomes and pump durability after implantation (1-5).

The miniaturized third-generation centrifugal pumps HeartWare HVAD (Medtronic, Dublin, Ireland; HeartWare International, Inc., Framingham, Mass, USA) and HeartMate 3 (Abbott, Illinois, USA; Thoratec, Pleasanton, CA, USA) are the two most commonly implanted systems in the contemporary era (1-7). Their design (Figure 1) has allowed clinicians to pioneer several alternate and less invasive implantation techniques to tackle a broad spectrum of clinical scenarios (6-10).

A brief review and discussion on alternative surgical techniques for both inflow and outflow cannula insertion, in the contemporary LVAD surgery era, are herein reported.

\section{Inflow cannula placement}

The most recent American Association for Thoracic Surgery (AATS)/International Society for Heart and Lung Transplantation (ISHLT) guidelines (6) and European Association for Cardiothoracic Surgery (EACTS) expert consensus document (7) recommend inflow cannula placement into the left ventricular (LV) apex anterior wall, about $2 \mathrm{~cm}$ lateral to the left anterior descending coronary artery, parallel to the interventricular septum towards the apical axis (connecting apex and mitral valve orifice) $(3,6,7)$. Angulating the cannula more than $7^{\circ}$ from this axis, as well as pushing the cannula too deep into the $\mathrm{LV}$ chamber, negatively influence intracavitary hemodynamics and $\mathrm{LV}$ unloading, increasing risk of pump thrombosis $(3,6,7)$.

Therefore, careful inflow insertion site planning 


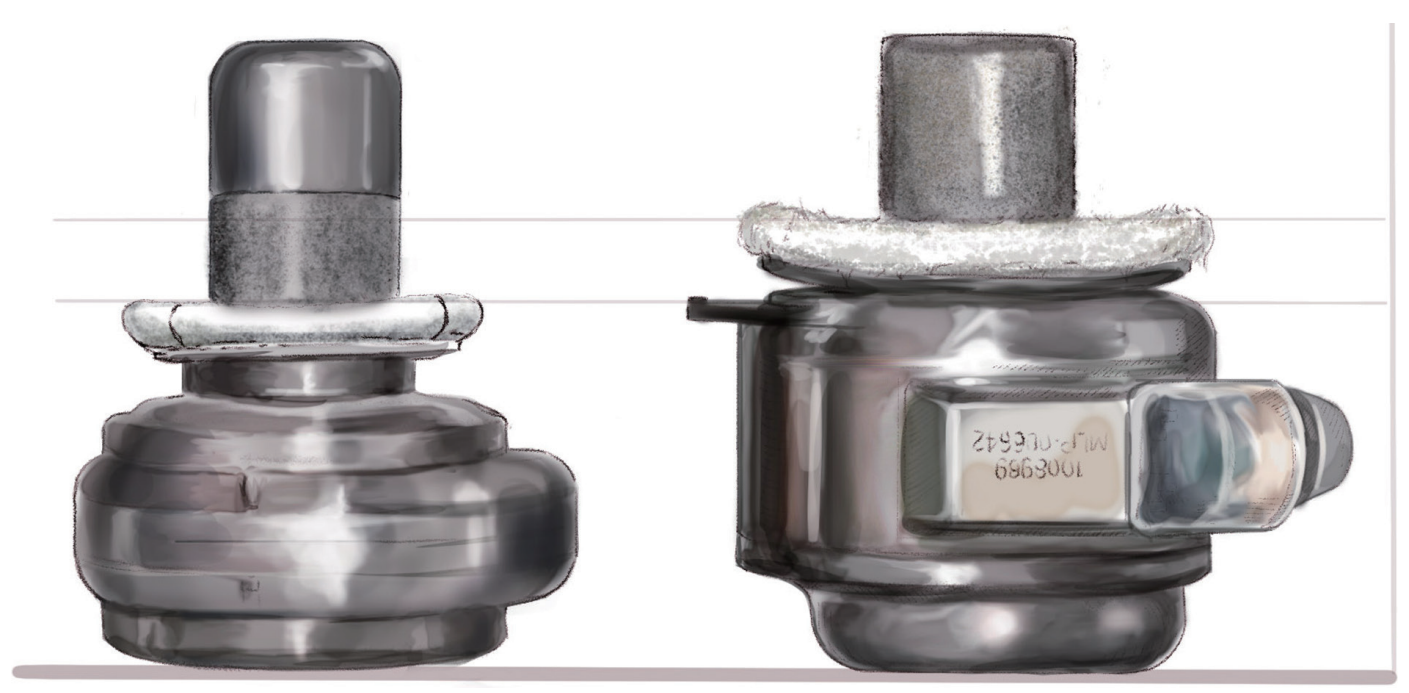

Figure 1 Profiles of current third-generation centrifugal pumps.

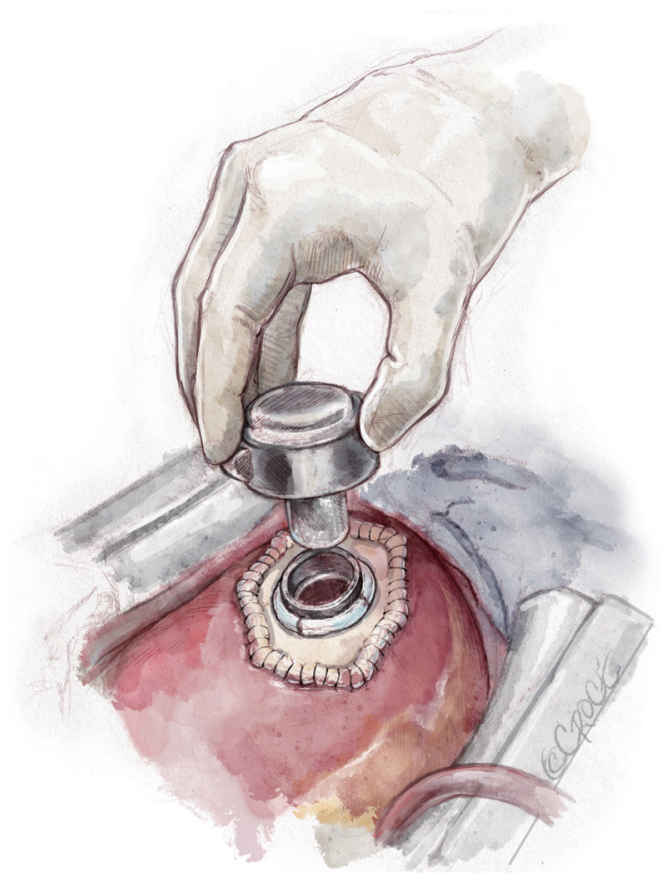

Figure 2 Left ventricular assist device insertion after concomitant left ventricular aneurysm reconstruction with a synthetic patch.

and proper device choice are crucial to obtain the best postoperative outcomes. However, small or restricted left ventricles are at higher risk of postoperative complications thus requiring alternative sites for cannulation and adoption of paracorporeal pulsatile pumps (11).

Direct access to the LV apex, as during sternal-sparing approaches, without heart dislocation, may facilitate placement of the inflow cannula without need of cardiopulmonary bypass (CPB) installation $(6,7)$.

The correct position of the sewing ring is identified under trans-esophageal echocardiographic (TEE) control, through finger or needle localization $(3,6,7)$. The sewing ring is fastened to the LV apex with, usually, twelve pledgeted prolene sutures, placed deeply into the myocardium; after a circular or X-shaped full-thickness ventriculotomy, myocardial coring is performed and the $\mathrm{LV}$ chamber is inspected and freed of thrombi or trabeculae crossing the inflow site if on CPB. The inflow cannula is inserted into the $\mathrm{LV}$, embedded in the sewing ring and rotated clockwise or counter-clockwise, according to the planned outflow anastomotic site (3,6-8).

Prior Dor procedure or concomitant LV aneurysm reconstruction represents a challenge because of pericardial adhesions, thin wall, apical calcification, apical thrombosis and restricted LV cavity $(6,7,12)$. If the residual capacity of the $\mathrm{LV}$ and the wall tissue thickness are sufficient to prevent suction, the site of previous ventriculotomy could be suitable for the inflow cannula; alternatively, the anastomosis could be performed to a synthetic patch (Figure 2), although at the expense of device stability and hemostasis $(6,7,12)$. In the case of restricted $L V$ and unviable walls, a left atrial inflow cannulation could be required and adoption of a paracorporeal pulsatile pump would be a solution.

The inferior $\mathrm{LV}$ wall may be considered as an alternative access site $(6,7,13)$ : severely dilated $\mathrm{LV}$ with impaired 


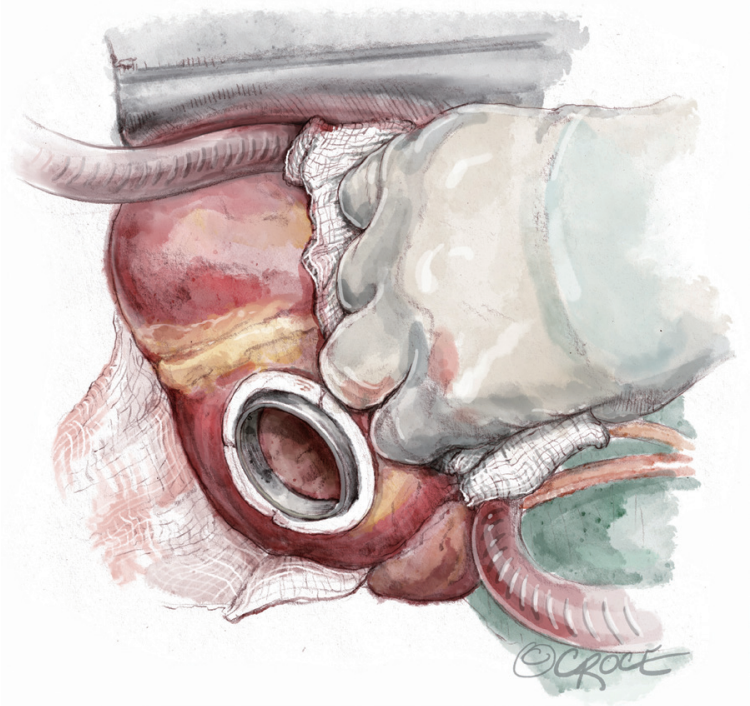

Figure 3 Sewing ring placement on the inferior left ventricular wall.

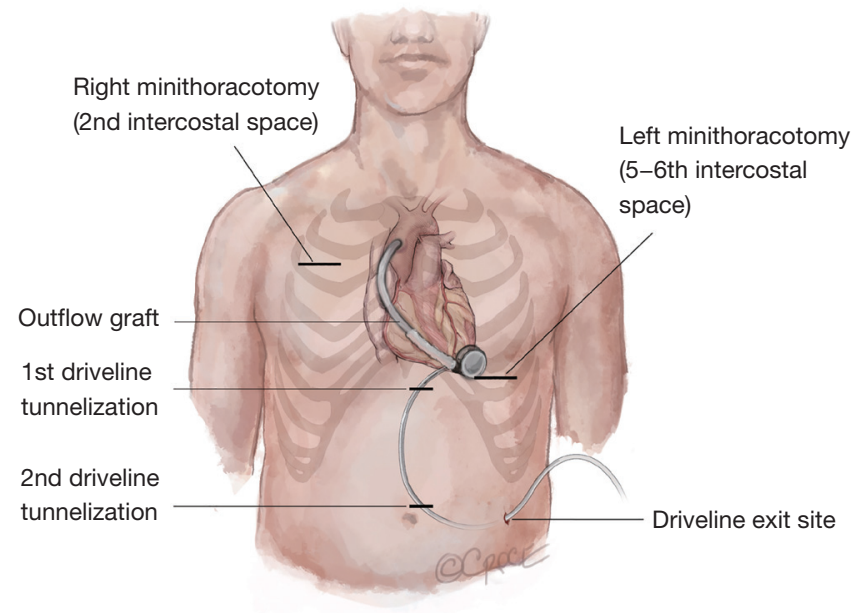

Figure 4 Intrapericardial tunneling scheme of outflow graft from the left side to the ascending aorta, during less invasive sternotomy-sparing approaches.

geometry, post-infarction LV apex aneurysm and narrowedchest patients may benefit from inflow cannula placement through the LV diaphragmatic wall (Figure 3). Technically, the $L V$ apex is lifted upwards and the sewing ring site is identified at "Frazier's point", which is located laterally to the posterior descending coronary artery, at the first third of the distance from the apex to the base of the heart, far away from the papillary muscles (13). The correct position and the absence of cannula obstructions may be checked with epicardial ultrasound or digital exploration during CPB. After coring, the inflow cannula is inserted along the short axis of the $\mathrm{LV}$, without reaching the interventricular septum. The pump is rotated with the outflow directed towards the right side of the heart, allowing outflow anastomosis to the ascending aorta $(6,7,13)$. However, this technique has currently been abandoned.

\section{Outflow graft positioning}

The recommended and preferred outflow site $(3,6,7)$ is the ascending aorta on the right curvature at about $2 \mathrm{~cm}$ above the sinotubular junction. After partial clamping of the aorta, anastomosis should be performed in an end-to-side fashion with a $5 / 0$ prolene running suture with $45^{\circ}$ angle. In the case of severe aortic calcifications a $4 / 0$ prolene suture may be of help. This allows physiological direct flow, reducing the hemodynamic burden on the root wall and on the aortic valve and, consequently, lowering the incidence of late aortic regurgitation $(3,6,7)$.

The course of the graft should be intrapericardial $(3,6,7)$ : during less invasive sternotomy-sparing approaches, the graft is tunneled from left side to the ascending aorta (6-10) (Figure 4). This may not always be a viable option in patients with previous cardiac surgery; with strong pericardial adhesions, an alternate site for graft anastomosis may be required.

The outflow line should run along the inferior right ventricle (RV) surface and laterally to the right atrium in order to avoid crossing the RV outflow tract. Care must be taken to avoid kinking or twisting of the outflow graft with the help of the longitudinal 'black' line markers. Graft length may be easily adjusted with clockwise or counterclockwise rotation of the centrifugal LVAD (6-10).

An alternative course of the outflow line via a less invasive approach and bridge to transplantation (BTT) strategy may be through the transverse sinus (Figure 5) $(6,7,14)$. This technique protects the LVAD graft from potential positional changes after sternal closure and enables easier and safer re-entry during the eventual heart transplantation. Once the transverse sinus has been identified and the membrane behind the aorta has been tunneled, a forcep is inserted through the sinus, from right to the left, and the outflow graft is pulled towards the standard anastomosis site on the ascending aorta. A Gore-Tex membrane covering the outflow is positioned in order to avoid strong adhesions. Some concerns have been reported regarding covering the 


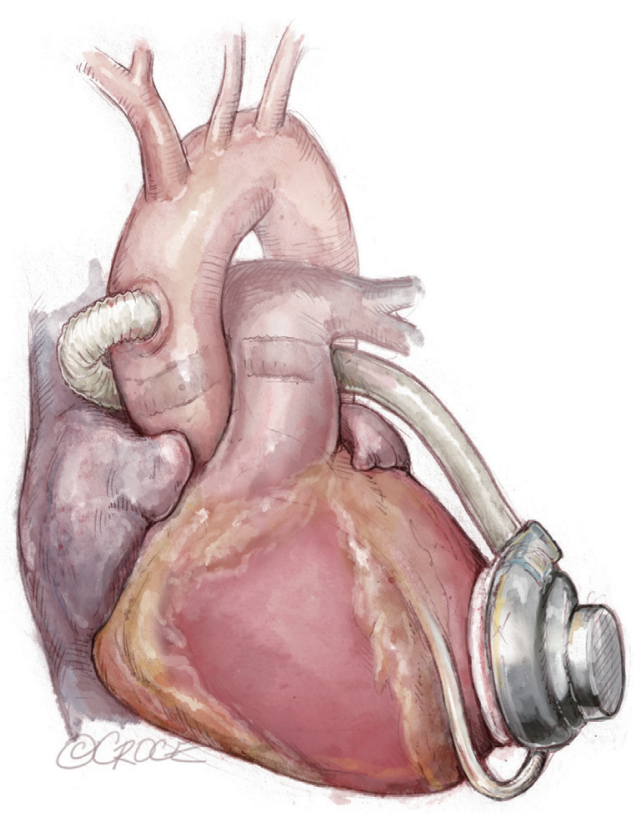

Figure 5 Outflow graft tunneling through the transverse sinus.

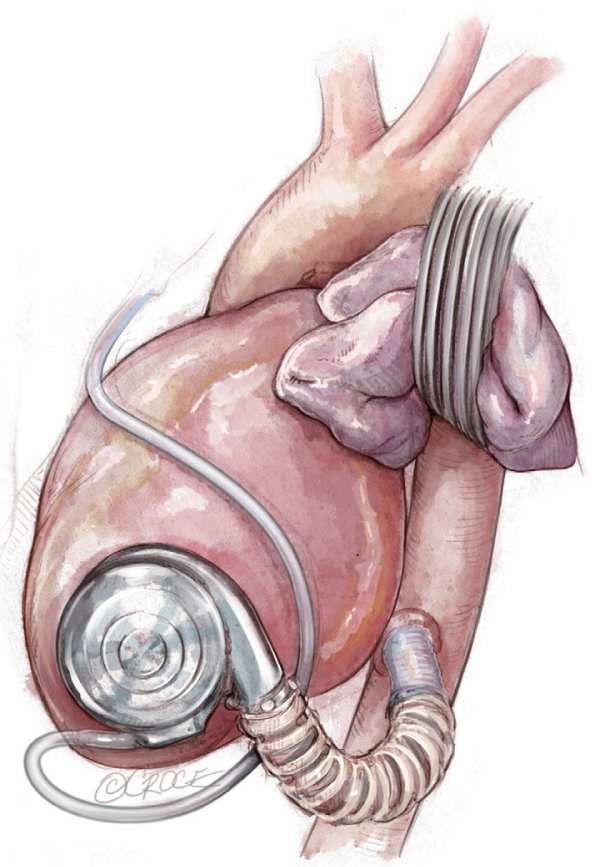

Figure 6 Outflow graft anastomosis to the descending aorta.

strain relief and outflow graft with polytetrafluoroethylene (PTFE): despite its 'anti-adherent' function, it could enhance thrombus formation between the graft and the PTFE itself, causing extrinsic obstruction of the outflow.
Thus a longitudinal incision of the PTFE membrane, at the time of outflow graft coverage, may be a solution $(6,7)$. However, in patients with previous cardiac surgery, this technique is not recommended because the transverse sinus is often stuck $(7,14)$.

In the case of heavy calcification or pseudoaneurysms, the ascending aorta is contra-indicated as an outflow graft anastomosis site $(13,14)$.

Alternative sites, such as the descending thoracic aorta, supra-celiac abdominal aorta, innominate artery or subclavian arteries, have been described with acceptable results $(6-10,15-17)$.

\section{Outflow graft anastomosis to the descending aorta}

The outflow graft anastomosis to the descending aorta is an integral part of 'lateral LVAD implantation' (Figure 6), which represents a viable technique in the case of chest re-entry, particularly in the presence of prior coronary artery bypass grafting (CABG) and/or valve replacement surgery $(6,7,15)$. After thoracotomy, the inferior pulmonary ligament has to be divided in order to make a pathway to the aorta. The descending aorta is exposed from the level of the pulmonary hilum to the diaphragm. CPB or Extracorporeal Life Support (ECLS) is then established, the coring process is performed, the LVAD secured and the outflow graft is measured and deaired. Off-pump surgery may be another option for LVAD implantation. The outflow is then buried in the left pulmonary fissure and anastomosed end-to-side to the aorta during lateral partial clamping.

The technique may have a number of advantages, including reduced surgical trauma and less risk of haemorrhage, protection of patent bypass grafts, avoiding mobilization of the apex and consequent ventricular rupture in cases with prior mechanical mitral valve implantation, as well as compatibility with off-pump operations (15). However, the clinical benefits of avoiding re-sternotomy have to be weighed against potential risk of retrograde flow from distal anastomosis sites, which may lead to aortic root thrombosis $(6,7,15)$.

\section{Outflow graft anastomosis to the supra-celiac abdominal aorta}

If the descending aorta displays heavy, diffuse calcification or atherosclerotic plaque, the supra-celiac abdominal aorta may be used for the outflow anastomosis site $(6,7,9)$ (Figure 7). A left subcostal incision is preferred in this 


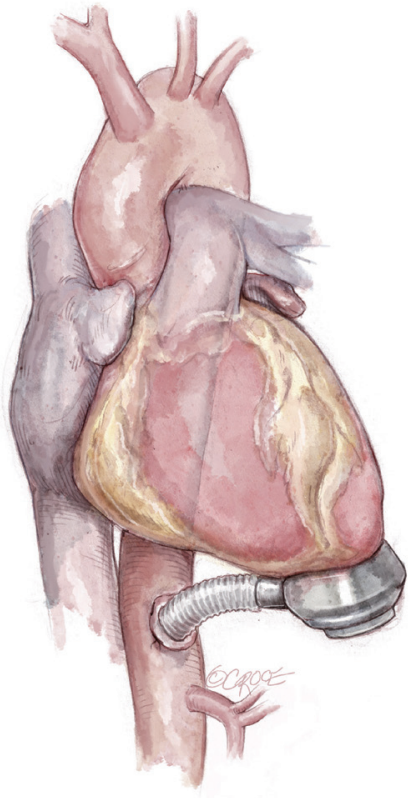

Figure 7 Outflow graft anastomosis to the supra-celiac abdominal aorta.

case. The aorta is exposed with extra-peritoneal access and the left diaphragm is divided to expose the heart. After femoral CPB is initiated, the LVAD is implanted on the diaphragmatic LV wall; the outflow graft is then anastomosed, in the traditional fashion. ECLS or offpump surgery may be other options for supra-celiac LVAD implantation.

\section{Outflow graft anastomosis to the innominate artery}

Outflow graft anastomosis to the innominate artery is optimal in BTT and is a good alternative in cases of hostile ascending or descending aorta $(6-9,16)$. During the standard sternotomy approach, the outflow graft runs along the RV profile, between the pericardium and the right atrium. It then passes over the superior vena cava and deep to the innominate vein to be anastomosed to the innominate artery (Figure 8). During minimally invasive approaches (upper hemi-sternotomy is the preferred access), the outflow may be tunneled either intrapericardially or, in redo cases, through the left pleural cavity $(6-9,16)$.

\section{Outflow graft anastomosis to the axillary artery}

Both left and right axillary arteries may be considered as outflow graft anastomosis sites (Figure 9) (6-10). Despite

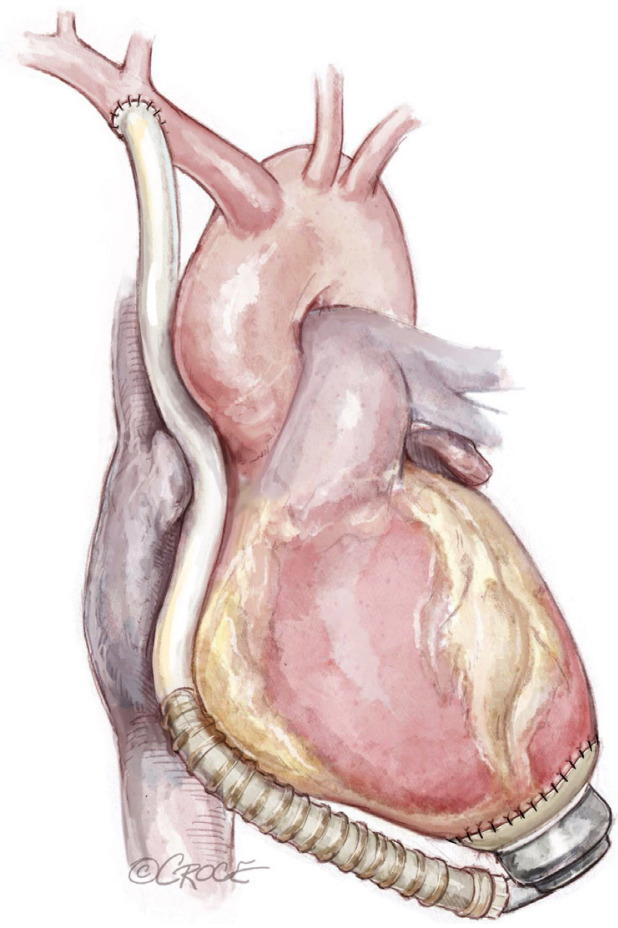

Figure 8 Outflow graft anastomosis to the innominate artery.

the advantages in the minimally invasive surgery setting, this choice presents three main technical issues requiring evaluation before LVAD implantation: the small caliber of axillary arteries, the need for intra-thoracic tunneling through an intercostal space for the outflow graft and the risk of left arm overflow or peripheral malperfusion.

After a subclavian or a deltopectoral incision, the axillary artery is exposed: usually, the small size of the vessel makes this technique compatible with devices in which the outflow vascular graft has a diameter no more than $10 \mathrm{~mm}$. The anastomosis may be performed in different ways according to axillary artery diameter: if the diameter is larger than $8 \mathrm{~mm}$, end-to-side or end-to-end anastomosis (combined with side anastomosis of distal axillary) can be used (7-10). Smaller arteries with a diameter less than $8 \mathrm{~mm}$ may require an $8 \mathrm{~mm}$ diameter prosthesis bridge $2-2.5 \mathrm{~mm}$ in length and a graft-to-graft anastomosis (7-10). The graft is protected with a ring-reinforced Gore-Tex membrane, preventing it from kinking and compression, and is tunneled at the first or second intercostal space. In order to avoid distal overflow, axillary artery bending is recommended: after the pump is started, bending is tightened until right and left radial pressures are balanced. Right axillary artery anastomosis was first described during HeartMate II implantation in 

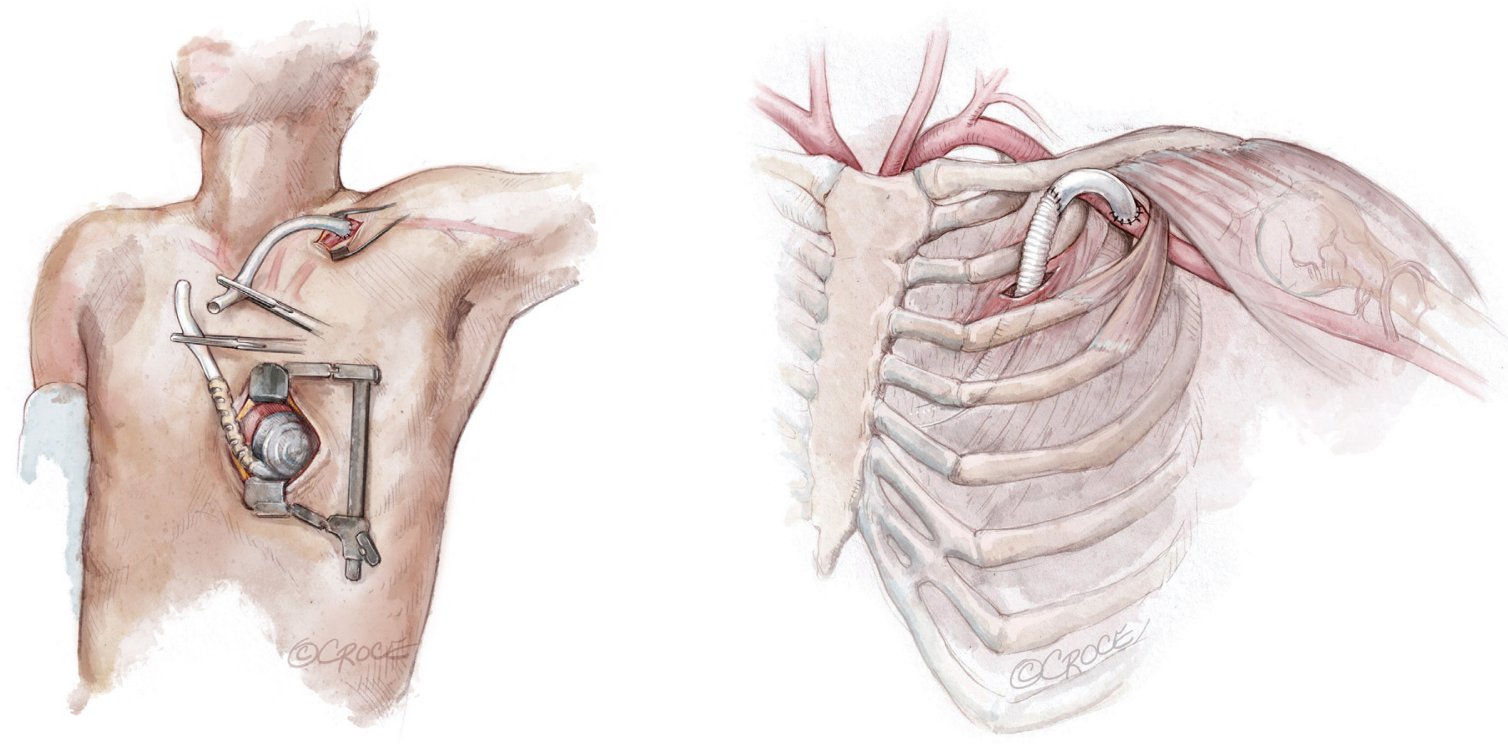

Figure 9 Scheme of outflow graft anastomosis to the left axillary artery, during less invasive sternotomy-sparing approaches.

the setting of heavily calcified ascending and descending aorta: in this case, the outflow is tunneled through the right hemidiaphragm, right pleural cavity and second intercostal space (6-10). Currently, HeartWare HVAD is the device of choice for such a surgical approach due to the smaller caliber (10 mm) outflow graft (6-10).

Despite being a well-accepted strategy, in minimally invasive surgery, arm swelling, movement impairment and neurological adverse events remain potential issues to be monitored.

\section{Biventricular assist device (BiVAD) setting}

After the first reports of the newer miniaturized LVAD systems, a fully implantable CF biventricular support has been discussed and judged feasible by the scientific community (17-20). Since 2010 several mechanical circulatory support centres have been advocating a technique which allows the use of two implantable centrifugal LVADs, HeartWare HVAD or HeartMate 3 as a biventricular assist system (BiVAD) (6,7,17-20) (Figure 10).

Patients who need biventricular long-term support either present primarily with severe chronic biventricular failure or have suffered secondary refractory RV failure after LVAD implantation, thus requiring additional long-term $\mathrm{RV}$ mechanical support $(6,7,17)$.

Currently, it is recommended that the right-sided pump be inserted into the right atrium or to the diaphragmatic RV wall $(7,17)$.

To provide a 'physiological' flow range of 3 to 6 liters per minute within a system speed setting of between 2,300 and 3,600 rpms, as usually set when the HVAD is used in terms of LVAD configuration, the afterload of the right system should be somehow increased. The Berlin team recommends reducing the outflow graft diameter to let the RVAD afterload reach the levels of the systemic circulation, artificially $(7,17)$. This can be done by reducing the graft diameter to approximately $5 \mathrm{~mm}$ in patients with normal and to $6-7 \mathrm{~mm}$ in patients with elevated pulmonary vascular resistance $(7,17)$. Graft reduction can be performed by sideclamping and narrowing the graft by placement of titanium clips. An Hegar bar is adopted for calibration. The length of outflow conduit narrowing should be about $30 \mathrm{~mm}$ thus influencing the right ventricular assist device (RVAD) afterload according to Hagen-Poiseuille law (17).

To reduce the length of the inflow cannula to be inserted into the right atrium, it is recommended to add, ideally, two Teflon felt tailored rings (up to $5 \mathrm{~mm}$ of thickness) to the original LVAD implantation ring $(7,17)$. If this is not available, hand-made rings of Dacron or Teflon felt velour can be tailored. Additionally, the pericardium should be cut down, laterally, preserving the phrenic nerve, and the pump body should be placed into the right pleural space and suspended by the incised pericardial wall which stays 


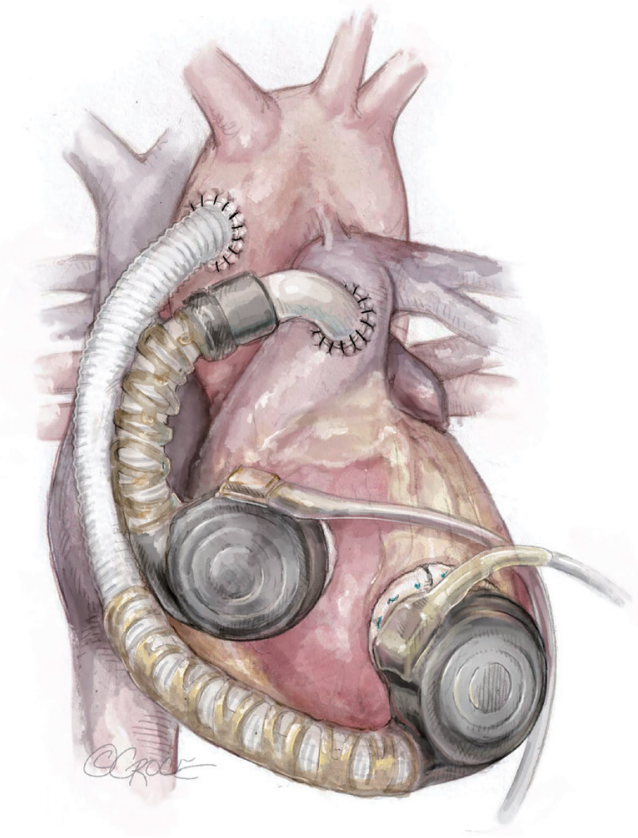

Figure 10 Biventricular assist device (BiVAD) setting.

between the right atrial surface and the device housing to facilitate an optimal position of the pump and avoid deep penetration of the inflow cannula into the right heart. The RVAD should be covered by a Gore-Tex membrane to avoid damage to the lung.

More than 400 CF-BiVADs have been implanted worldwide (1,2,17-20), even in the pediatric population (18) with acceptable success rates compared to paracorporeal pulsatile BiVADs and total artificial heart (TAH) support.

The surgery for two pulsatile pumps for biventricular long-term support leads to an extensive and traumatic operation both for pump housing location and penetrating cannulae positioning (e.g., Berlin Heart Excor, Berlin Heart GmbH, Berlin, Germany). Thus, the risk of bleeding and/ or infection is high $(7,17)$.

An implantable CF BiVAD system should be considered advantageous in terms of greater comfort and quality of life. The two systems (both HeartWare HVAD and HeartMate 3) run completely silently, and even if patients have to carry a couple of controllers and four batteries, they report a higher degree of freedom and mobility (7,17-20). Future developments of the current centrifugal pumps by industry focusing on the BiVAD cohort will provide significant improvements in patients' quality of life. TAH support by utilizing two CF radial pumps has been reported but still remains anecdotal (20).

\section{Conclusions}

Less invasive and alternative surgical techniques for longterm contemporary LVAD placement are technically feasible and reproducible with contemporary centrifugal devices.

Novel surgical options for inflow and outflow cannula placement may increase the number of high-risk surgical patients who may benefit from CF-LVAD therapy.

Further scrutiny is needed to clarify the potential advantages and disadvantages of these novel techniques on CF-LVAD patient outcomes.

\section{Acknowledgments}

Funding: None.

\section{Footnote}

Conflicts of Interest: The authors have no conflicts of interest to declare.

Open Access Statement: This is an Open Access article distributed in accordance with the Creative Commons Attribution-NonCommercial-NoDerivs 4.0 International License (CC BY-NC-ND 4.0), which permits the noncommercial replication and distribution of the article with the strict proviso that no changes or edits are made and the original work is properly cited (including links to both the formal publication through the relevant DOI and the license). See: https://creativecommons.org/licenses/by-nc-nd/4.0/.

\section{References}

1. Teuteberg JJ, Cleveland JC, Cowger J. The Society of Thoracic Surgeons Intermacs 2019 Annual Report: The Changing Landscape of Devices and Indications. Ann Thorac Surg 2020;109:649-60.

2. de By TMMH, Mohacsi P, Gahl B, et al. The European Registry for Patients with Mechanical Circulatory Support (EUROMACS) of the European Association for Cardio-Thoracic Surgery (EACTS): second report. Eur J Cardiothorac Surg 2018;53:309-16.

3. Slaughter MS, Rogers JG, Milano CA, et al. Advanced heart failure treated with continuous-flow left ventricular assist device. N Engl J Med 2009;361:2241-51.

4. Strueber M, O'Driscoll G, Jansz P, et al. Multicenter evaluation of an intrapericardial left ventricular assist 
system. J Am Coll Cardiol 2011;57:1375-82.

5. Mehra MR, Naka Y, Uriel N, et al. A fully magnetically levitated circulatory pump for advanced heart failure. $\mathrm{N}$ Engl J Med 2017;376:440-50.

6. Kirklin JK, Pagani FD, Goldstein DJ, et al. American Association for Thoracic Surgery/International Society for Heart and Lung Transplantation guidelines on selected topics in mechanical circulatory support. J Heart Lung Transplant 2020;39:187-219.

7. Potapov EV, Antonides C, Crespo-Leiro MG, et al. 2019 EACTS Expert Consensus on long-term mechanical circulatory support. Eur J Cardiothorac Surg 2019;56:230-70.

8. Maltais S, Anwer LA, Tchantchaleishvili V, et al. Left lateral thoracotomy for centrifugal continuous-flow left ventricular assist device placement: an analysis from the Mechanical Circulatory Support Research Network. ASAIO J 2018;64:715-20.

9. El-Sayed Ahmed MM, Aftab M, Singh SK, et al. Left ventricular assist device outflow graft: alternative sites. Ann Cardiothorac Surg 2014;3:541-5.

10. McGee E, Danter M, Strueber M, et al. Evaluation of a lateral thoracotomy implant approach for a centrifugalflow left ventricular assist device: The LATERAL clinical trial. J Heart Lung Transplant 2019;38:344-51.

11. Chivukula VK, Beckman JA, Prisco AR, et al. Small Left Ventricular Size Is an Independent Risk Factor for Ventricular Assist Device Thrombosis. ASAIO J 2019;65:152-9.

12. Kervan U, Unal EU, Sert DE, et al. Heartware left ventricular assist device implantation combined with

Cite this article as: Loforte A, Bottio T, Attisani M, Martin Suarez S, Tarzia V, Pocar M, Botta L, Gerosa G, Rinaldi M, Pacini D. Conventional and alternative sites for left ventricular assist device inflow and outflow cannula placement. Ann Cardiothorac Surg 2021;10(2):281-288. doi:10.21037/acs-2020cfmcs-28 surgical ventricular reconstruction. Exp Clin Transplant 2019; 17:278-80.

13. Gregoric ID, Cohn WE, Frazier OH. Diaphragmatic implantation of the HeartWare ventricular assist device. J Heart Lung Transplant 2011;30:467-70.

14. Hanke JS, Rojas SV, Cvitkovic T, et al. First results of HeartWare left ventricular assist device implantation with tunnelling of the outflow graft through the transverse sinus. Interact Cardiovasc Thorac Surg 2017;25:503-8.

15. Ozbaran M, Yagdi T, Engin C, et al. Left ventricular assist device implantation with left lateral thoracotomy with anastomosis to the descending aorta. Interact Cardiovasc Thorac Surg 2018;27:186-90.

16. Hanke JS, Rojas SV, Martens A, et al. Minimally invasive left ventricular assist device implantation with outflow graft anastomosis to the innominate artery. J Thorac Cardiovasc Surg 2015;149:e69-70.

17. Eulert-Grehn JJ, Lanmüller P, Schönrath F, et al. Two implantable continuous-flow ventricular assist devices in a biventricular configuration: technique and results. Interact Cardiovasc Thorac Surg 2018;27:938-42.

18. Stein ML, Yeh J, Reinhartz O, et al. HeartWare HVAD for biventricular support in children and adolescents: The Stanford Experience. ASAIO J 2016;62:e46-51.

19. Lavee J, Mulzer J, Krabatsch T, et al. An international multicenter experience of biventricular support with HeartMate 3 ventricular assist systems. J Heart Lung Transplant 2018;37:1399-402.

20. Lebreton G, Mastroianni C, Amour J, et al. Implantation of Two HVADs Used as a Total Artificial Heart: A New Approach. Ann Thorac Surg 2019;107:e165-e167. 